their impact on SLICC/ACR damage index (SDI) and disease outcome.

Results We detected one or more SDI scores in $77.87 \%$ of patients. Patients with disease duration of more than 10 years and subjects diagnosed at age above 40 had significantly higher SDI values. The most frequent damages were valvulopathies, cognitive dysfunction, angina pectoris and venous thrombosis. Higher cumulative glucocorticoid dose increased SDI, while chloroquin treatment was favourable for patients. Male gender, elevated SDI scores and higher cumulative doses of glucocorticoids increased mortality risk. Our data confirmed that disease duration, age at diagnosis, chronic high-dose glucocorticoid therapy have significant effects on the development of chronic organ damage. Higher SDI score is characterised with worse survival ratios. The most common chronic organ damages affected the cardiovascular or neuro-psychiatric system.

Conclusions As long-term survival in SLE improves, it becomes increasingly important to identify the determinants of chronic organ damage. Most of the chronic organ damage occurs in the cardiovascular and the neuropsychiatric systems, thus regular follow-up, screening and adequate therapy are essential for the best clinical outcome.

\section{PS7:147 CLINICAL EXPERIENCE OF BELIMUMAB TREATMENT IN CLINICAL PRACTICE OF SLE PATIENTS}

${ }^{1} \mathrm{M}$ de la Rubia Navarro, ' $\mathrm{K}$ Arevalo Ruales, 'I Chalmeta Verdejo, 1,2J Ivorra Cortes, ${ }^{2} \mathrm{~S}$ Cerda Checa, ${ }^{1} \mathrm{C}$ Alcañiz Escandell, II Canovas Olmos, ${ }^{1} \mathrm{CM}$ Feced Olmos, ${ }^{1} \mathrm{JJ}$ Fragio Gil, ${ }^{1} R$ Gonzalez Mazario, 'L Gonzalez Puig, 'E Grau Garcia, 'E Labrador Sanchez, II Martinez Cordellat, ${ }^{1} \mathrm{C}$ Najera Herranz, ${ }^{1} \mathrm{R}$ Negueroles Albuixech, ${ }^{1} \mathrm{JE}$ Oller Rodriguez, ${ }^{1} \mathrm{FM}$ Ortiz Sanjuan, ${ }^{1}$ E Vicens Bernabeu, ${ }^{1,2} \mathrm{JA}$ Roman Ivorra. ${ }^{1}$ Rheumatology Department, HUP La Fe, Valencia, Spain; ${ }^{2}$ Medical School, UCV, Valencia, Spain

\subsection{6/lupus-2018-abstract.190}

Purpose To study the clinical safety and adverse events (AEs) of Belimumab treatment in routine clinical practice in SLE patients.

Methods Retrospective observational study in which data from patients diagnosed of SLE according to SLICC 2012 criteria treated with intravenous Belimumab therapy (Initial: 3 doses $10 \mathrm{mg} / \mathrm{kg}$ IV every 14 days and maintenance dose: $10 \mathrm{mg} / \mathrm{kg}$ IV every 28 days) were collected. Analytical data of serological profile, clinical manifestations at the onset of the disease and at present, concomitant immunosuppressant and AEs (grouped in non-infectious, infectious, and infusion/hypersensitivity reactions) from July 2012 to September 2017 were collected.

Results A total of 15 patients [13 women (86.6\%), median (SD) age 32 (8.34) and age at diagnosis 20.17 (11.5) years] were included. Median follow-up was 20 months (range, 161). Serologic Activity and clinical manifestation are shown on table 1.

Discontinuation of Belimumab therapy was observed in 4 patients after a 1, 16, 13, and 61 months of follow-up respectively. One patient discontinued voluntary after 61 months of treatment due to desire for pregnancy. The other three patients discontinued by itchy skin lesions, primary pulmonary hypertension and peripheral venous insufficiency.

21 AEs were reported (19 infectious and 2 non-infectious), and 18 occurred in patients with more than 12 months of follow-up. No infusion-related reactions were observed.
All patients received concomitant immunosuppressant therapy (hydroxychloroquine in 8, mycophenolate mofetil in 6, azathioprine in 4, and methotrexate in 2). 12 patients were receiving simultaneous glucocorticoid treatment. We observed a significant decrease in the mean daily prednisone dose over time $(8.0 \mathrm{mg} /$ day at the beginning to $5.3 \mathrm{mg} /$ day at the end of study).

Conclusions In conclusion, our data confirm the safety of Belimumab therapy in SLE patients. Overall, 4 (20\%) patients discontinued treatment due to AEs and in one additional patient (7\%) treatment was stopped due to pregnancy. Reduction of disease activity was observed in 12 (80\%) of our patients. Finally, the significant decrease of prednisone dose is associated to and additional reduction in steroid-related AEs and with an increase in patient's quality of life.

\begin{tabular}{|c|c|}
\hline & $\begin{array}{l}\text { SLE patients } \\
\mathrm{N}=15\end{array}$ \\
\hline $\begin{array}{l}\text { Clinical manifestations at the onset: } \\
\text { Musculoskeletal } \\
\text { Mucocutaneous } \\
\text { Renal } \\
\text { Cytopenias }\end{array}$ & $\begin{array}{l}10(66.6 \%) \\
10(66.6 \%) \\
7(46.6 \%) \\
5(33.3 \%)\end{array}$ \\
\hline $\begin{array}{l}\text { Clinical manifestations at the end of follow-up: } \\
\text { Musculoskeletal } \\
\text { Cytopenias } \\
\text { Mucocutaneous } \\
\text { Renal }\end{array}$ & $\begin{array}{c}11(73.3 \%) \\
11(73.3 \%) \\
10(66.6 \%) \\
3(20 \%)\end{array}$ \\
\hline $\begin{array}{l}\text { Serological activity (defined as reduction level } \\
\text { of } \mathrm{C} 3 / \mathrm{C} 4 \text { and/or high level of anti-ds DNA) }\end{array}$ & $12(80 \%)$ \\
\hline
\end{tabular}

\section{PS7:148 THE RELATIONSHIP BETWEEN HYPOCOMPLEMENTEMIA AND HAEMATOLOGICAL INVOLVEMENT IN ALBANIAN SLE PATIENTS}

M Jordhani, V Duraj, D Ruci, A Kollcaku. Uhc Mother Teresa, Tirana, Albania

\subsection{6/lupus-2018-abstract.191}

Introduction Haematological involvement in Systemic Lupus Erythematosus (SLE) is common in patients with SLE. On the other hand, one of the most important variables that helps in disease activity and severity is complement serum level.

Objectives The aim of this study was to evaluate the relationship between haematological involvement in Systemic Lupus Erythematosus patients and hypocomplementemia.

Methods This is an observational study where 62 patients with SLE haematological involvement were included. All the patients were followed-up at UHC Mother Teresa, Tirana, Albania. All clinical and laboratory data were evaluated, gathered and analysed at our University clinic. It was evaluated especially the level of complement and the complete blood count in order to achieve the data needed for this study.

Results It was found that from 62 patients with SLE and haematological involvement, 11 patients were found with only one series affected (anaemia, leucopenia, thrombocytopenia), 10 patients were found with bicytopenia, and 41 were found with pancytopenia. After evaluating the complement levels, hypocomplementemia was found in 2 patients (18.2\%) from the first group, in 8 patients (80\%) from the second group, and 38 patients $(92.6 \%)$ from the third group. 